July - December 2018

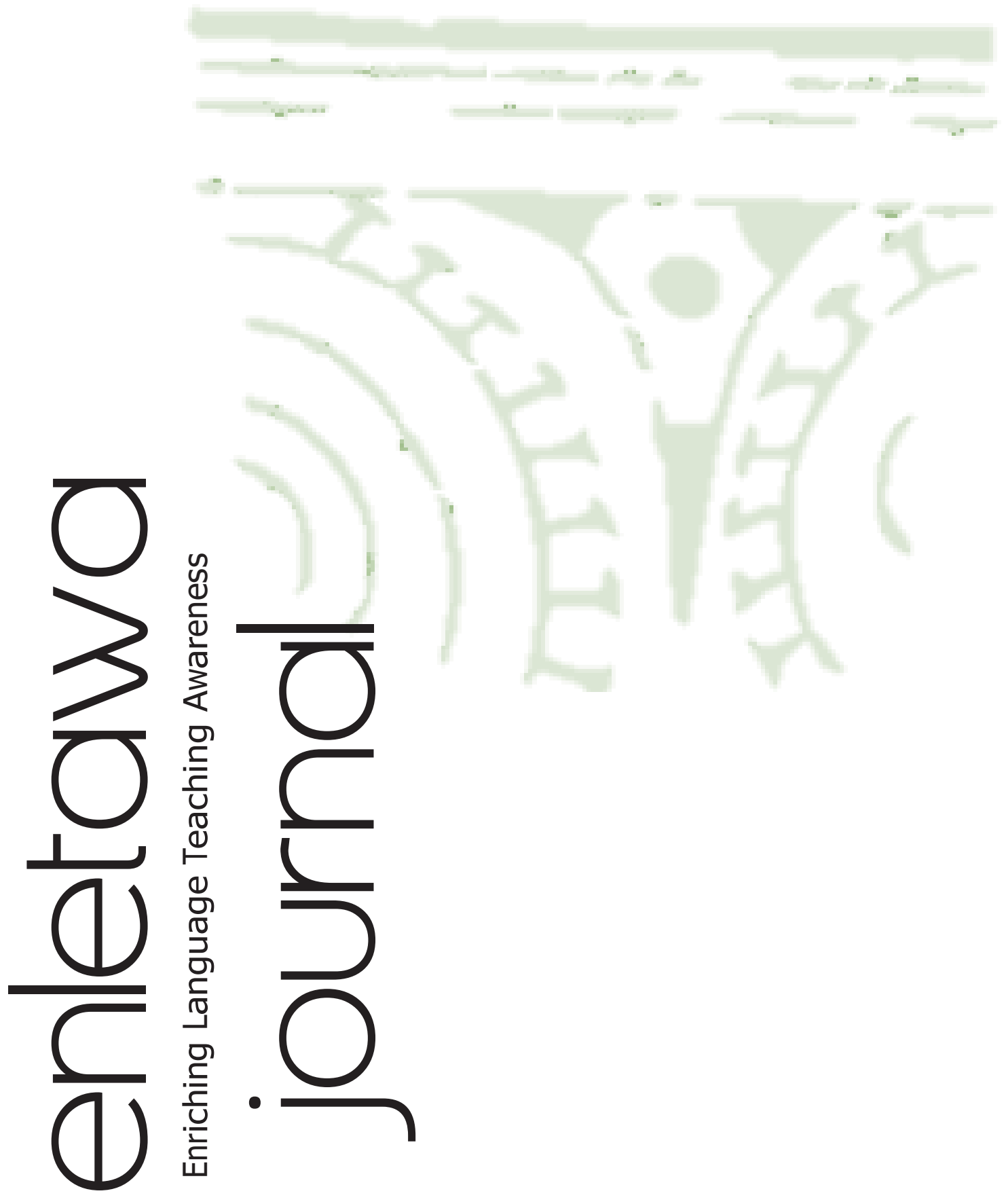

Vol. 11, No. 2
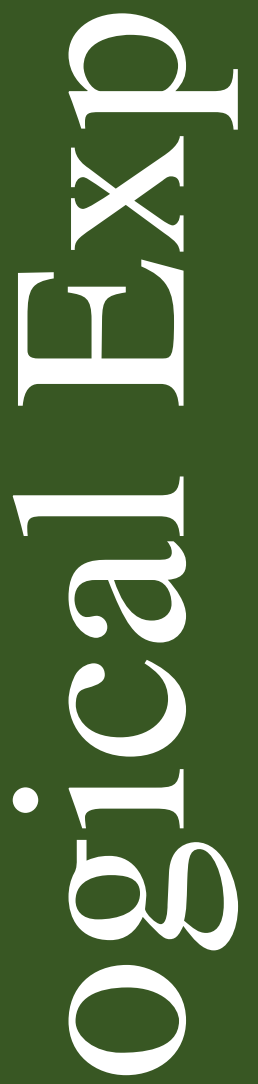


\title{
Sesión de Retórica: Espacio de Encodificación ${ }^{1}$ Rhetoric Session: Space of Encoding
}

\author{
César Augusto Romero Farfán ${ }^{2}$ \\ Universidad Pedagógica y Tecnológica de Colombia \\ cesar.romero@uptc.edu.co
}

\section{Received: November 12, 2018}

Accepted: November 26, 2018

How to cite this article (APA, ${ }^{\text {th }}$ ed.): Romero Farfán, C. A. (2018). Sesión de retórica: Espacio de encodificación. Enletawa Journal, 11(2), 47-56.

\section{Resumen}

El tema de este artículo es "Sesión Interuniversitaria de Retórica". Proviene de una estrategia pedagógica consistente en un grupo de estudiantes que presenta, persuasivamente, teorías, en este caso lingüísticas, ante un grupo de coetáneos, para intentar interiorizarlas. Se puede aplicar, también, como estrategia pedagógica, en distintas clases o cursos para fortalecer capacidades de análisis, memorización, documentación, orales, escritas, de convicción y de persuasión de discentes de la educación básica o de la superior. El escrito puede ampliarse, por ejemplo, para establecer cómo una sesión interuniversitaria de retórica consolida el trabajo en equipo. Finalmente, se concluye sobre cómo la sesión de retórica es, sin duda, un espacio encodificador de actitudes y de conocimiento.

Palabras claves: Sesión de retórica, encodificación, lingüística de la lengua, dicotomías lingüísticas

\footnotetext{
${ }^{1}$ Pedagogical Experience.

${ }^{2}$ César Augusto Romero Farfán is a full-time professor in the School of Languages at the Universidad Pedagógica y Tecnológica de Colombia. He holds a Bachelor's in Education with a focus in Spanish and French. Additionally, César has a master's in Lingüística Hispánica, Investigación y Docencia. His areas of expertise include language, mother tongue, and communication.
} 


\begin{abstract}
The topic of this article is an "Interuniversity Session of Rhetoric". It comes from a pedagogic strategy consisting of a group of students that persuasively presented linguistic theories to a group of contemporaries to try to internalize them. It is possible to apply this strategy in different classes or courses to strengthen analysis, memorization, documentation, oral and written skills, and the ability of conviction and persuasion in basic education or high education. The writing can be extended, for example, to establish how an interuniversity session of rhetoric consolidates teamwork. Finally, this article concludes on how the session of rhetoric is, definitely, a space to encode attitudes and knowledge.
\end{abstract}

Keywords: Session of rhetoric, encoding, linguistics of the language, linguistic dichotomies. 


\section{Liminar}

El presente artículo pretende responder el interrogante: ¿cuál es la utilidad de la Sesión Interuniversitaria de Retórica, colegida a partir de la socialización, también interuniversitaria, de algunos contenidos propios de la Linguística, en el contexto de un plan de estudios de Idiomas Modernos, para reflexionar sobre tal Sesión de Retórica, como espacio de encodificación?

Se origina en una estrategia pedagógica utilizada, por más de 10 años, en la asignatura de Lingüística, como espacio académico interactivo de encodificación en el que un grupo de discentes, con base en un proceso teóricopráctico: documentación, exposición, seminario alemán, interiorizaciónencodificación, coevaluación y autoevaluación presentan, retóricamente, algunos temas propios de la historiografía de la ciencia del lenguaje, ante un grupo de pares de otras universidades. Se trata de socializar, con ánimo persuasivo, contenidos y teorías propios de la ciencia del lenguaje, ante un auditorio, generalmente, nutrido e interuniversitario, gracias a la ventaja de una ciudad, como Tunja, de tener una de las universidades públicas más importantes de Colombia, en general, y del oriente colombiano, en particular, y otras Instituciones de educación superior privadas, de gran relevancia en el contexto local, regional, nacional e internacional.

$\mathrm{Su}$ aplicación es posible en un contexto pedagógico que busque fortalecer, en los discentes, capacidades de habla, de oratoria, de convicción, de persuasión y fortalecerlos en lo que a expresión oral académica y fundamentada se refiere. Esto significa que lo escrito aquí se puede proyectar a la mayoría de contenidos curriculares de la Educación Básica y de la Superior.

El objetivo general de estas líneas es el de realzar la utilidad de la Sesión Interuniversitaria de Retórica, como espacio, momento, actividad, labor y estrategia que busca socializar contenidos desde la Lingüística y llevarlos hasta el momento de la encodificación, entendida como un conocimiento retenido que se evoca para hacerlo práctico o para fundamentar una praxis sobre el mismo.

Una de las dificultades más apremiantes, en relación con este escrito, es la independencia de ciertos conceptos; es decir, la autonomía con que se los ha fundamentado. Ello se debe al hecho de que los mismos son un medio y no un fin de los resultados aquí esbozados.

Finalmente, este escrito se componente de una fundamentación teórica breve; una metodológica y unos corolarios. En el aparte metodológico se elucida y explica cada una de las etapas propias del proceso Sesión Interuniversitaria de Retórica. Se concluye con las ventajas de la Sesión de Retórica, como herramienta potenciadora del aprendizaje y mediadora del logro de profesionales más humanos, conscientes y apasionados con su labor.

Palabras claves: sesión de retórica encodificación - lingüística de la lengua dicotomías lingüísticas.

\section{Fundamentación Teórica}

Teóricamente, este documento parte de la ciencia del lenguaje humano: la Lingüística. Como bien se sabe, esta ciencia se consolida en 1916 con el "Curso de Lingüística general", de Ferdinand de Saussure, obra escrita por sus estudiantes: 
Charles Bally y Albert Sechehaye, quienes contaron con la colaboración de su esposa $\mathrm{y}$, principalmente, de Albert Riedlinger. (V. Mahecha, 2005). En la cátedra de Linguística, impartida en la UPTC, se parte de Saussure porque sus discernimientos son vitales para entender la ciencia del lenguaje actual. Se intenta interiorizar en los discentes, entre otras nociones, las de dicotomía lingüística; diacronía, sincronía, signo lingüístico; lenguaje, lengua y habla; niveles de análisis lingüístico; fonema, morfema; semiología y símbolo (cfr. Bernárdez, 1999) que, a la vez, le aportan aforismos de trascendencia a las nociones de primera y segunda articulación y metalingüística de la lengua, fundamentales, hoy, si de elucidar el lenguaje como exclusivo de la especie humana se trata. (Cfr. Martinent, 1970; Bernárdez, 1993; Veyrat, 1999; López, 1999; COGRED, 2016-2017).

En síntesis se define la Lingüística, como el estudio científico del lenguaje humano, aunque se les recuerda, a los alumnos, que los conceptos de lengua y de habla son prioritarios, también, porque se toman como objeto de estudio en las principales Escuelas Linguiísticas del siglo $\mathrm{XX}$. Incluso, es complejo que el hablante discierna las diferencias, debido a que, en general, se habla, por ejemplo de lenguaje oral y escrito, cuando se debería referir lengua oral y escrita.

Con lo anterior, y para fines propios de este opúsculo, la ciencia del lenguaje se yuxtapone a planteamientos y aforismos en relación con la lectura y con la escritura. Bien se sabe que quienes llegan y permanecen en la Universidad tienen dificultades, cada vez más notorias, para leer y para escribir. Como ello es axiomático, se trabaja con la convicción de que la lectura y la escritura deben constituirse en tareas socialmente ineluctables porque leer y escribir, precisamente, son fundamentales en todo horizonte cultural, vital, humano, humanístico y universal -universitarioporque quien lee y lo registra por escrito es persona notable, dado que leer y escribir son verbos reflexivos: leerse, escribirse y nos ayudan a comprendernos, a interpretarnos, a interactuar con nosotros mismos -antes que con los demás- y a evocarnos (cfr. Torres Cruz, 2012, pp. 224-225).

Precisamente, con la lectoescritura se concreta, entre varias, una tarea fundamental al reflexionar sobre el lenguaje, la lengua y el habla: leer, en donde se lleva a cabo la labor de comprender, interpretar, retener y evocar (véase Santiago y Otros, 2006) y escribir, quehacer trabajado, al tenor de Cassany (1999), como un verbo transitivo porque no se escribe per se, se escribe con el (él), para y por el Otro.

Una vez los discentes han encodificado -entendido aquí, en primera instancia como utilizar el conocimiento interiorizado con algún fin vital- los contenidos por socializar durante la Sesión de Retórica, ésta se define como un espacio, interuniversitario en este caso, en el que debe convencerse, primero, persuadirse, luego, a un grupo de concurrentes coetáneos con los estudiantes. Adelante, se enumerará algunas formas de persuasión.

Para los romanos, parafraseando a Rosenblat y Sanabria (1968, pág. 98), la retórica era copiosa o ampulosa y concreta o de sacudidas bruscas. El segundo estilo retórico se caracteriza por el conceptismo; en el caso presente, no se trata, como lo apuntaban los conceptistas, de sacrificar un mundo por pulir un verso sino, por analogía, de comunicar una teoría 
lacónica, compleja, abstracta y exclusiva, por medio de una práctica fundamentada en la palabra que afecta los sentidos, que llega a ubicarse en la mente del interlocutor -público- por un buen tiempo, que llega a moverlo a la acción académica -leer más, escribir mejor- y que se puede entender sin necesidad de pertenecer a élites curriculares.

Por tanto, la Retórica se desprende de la Oratoria, se transfiere con la lectura consuetudinaria, y se registra merced a la escritura -de un guion sucinto-, especialmente, la expositiva y la argumentativa, cuyo fin último es el de la persuasión. Finalmente, todo se consolida en una presentación bibliográfica o en una dramatización, en un parafraseo musical, o en un coro poético, o en intervenciones ordenadas, $\mathrm{o}$ en imitaciones, $\mathrm{o}$ en imitaciones de medios masivos, o en remedo de la manera como tales medios atrapan a sus espectadores; en fin, en trabajos lingüísticos en los que la palabra, yuxtapuesta a necesarios componentes paralingüísticos y extralingüísticos, se convierte en un medio y en un fin de persuasión.

Aclárase que, además de los temas lingüísticos mencionados (cfr. supra), los alumnos pueden echar mano de las funciones del lenguaje y, dentro de ellas, de la metalingüística y de la estética, lo que permite un acercamiento al carácter prevaricador del lenguaje (véase López, citado por Veyrat, 1999). Este hecho, exclusivo de la especie humana y del lenguaje como facultad humana $y$ humanizadora, es supremamente exitoso como fundamento de la Sesión de Retórica cuando los estudiantes, grupalmente, despliegan creatividad y logran la admiración, a veces imitativa, de su público.
En fin, con Contreras (2015, citado por Cardoso y Castañeda, 2017) y Torres C. (2017), entre importantes estudiosos de la lectura y de la escritura, y de la oratoria en el marco de procesos orales de litigación con prospección holística, respectivamente, la ciencia del lenguaje y su respectiva Sesión de Retórica se convierte en una espacio propicio para consolidar la calidad educativa y social tan necesaria en el actual estado de cosas que se vive, políticamente, en lo local, lo regional, lo nacional y lo internacional.

\section{Fundamentación Metodológica}

La técnica principal para la recolección de la información ha sido la elicitación, y la observación semiestructurada y estructurada. El autor de estas líneas es el profesor de Lingüística (en los niveles I y II) de los sujetos de investigación, en el contexto de un currículo de Idiomas Modernos. Acopiando su experiencia en la organización, planeación, concreción y puesta en escena de la Sesión de Retórica por varios años, desde el inicio del semestre académico, el profesor pone en conocimiento de sus alumnos la forma de evaluar y de calificar el Curso. Precisamente, uno de los ejes de tal proceso se denomina: Sesión de Retórica. Generalmente, los alumnos aceptan un $25 \%$, de un $100 \%$, como valor de la dicha Sesión. Sin embargo, en otras ocasiones, el porcentaje mencionado aumenta. Probablemente, depende de la experiencia que, semestre tras semestre, se comunican los futuros Licenciados.

Recuérdese que, en la UPTC, los semestres se evalúan mediante dos $50 \%$, de 8 semanas cada uno. En este punto, suele presentarse la Sesión de Retórica ante la comunidad de pares, casi siempre, 
durante el segundo 50\%. Sin embargo, el proceso de su planeación y preparación comienza desde el inicio del Semestre académico. Tal proceso, acorde con lo escrito en el liminar de este documento, se divide en una serie de etapas que, mediante un ejemplo, se explican a continuación:

DOCUMENTACIÓN: el profesor elige el tema para Sesión de Retórica. Los alumnos comienzan, de acuerdo con la bibliografía del programa de la opción temática de Lingüística, a leer autores y perspectivas teóricas sobre dicho tema. De hecho, deben elegir algunas líneas sobre el particular y, basados en un esquema, presentar una exposición sobre la que, previamente, entregan un guion sucinto (se le denomina así porque el fundamento teórico puede, incluso, fotocopiarse, siempre y cuando se le añada su referencia bibliográfica completa. Si se trata de una página web, se advierte su valía cuando existe un autor o una institución que responda por ella. Así mismo, cuando tenga una fecha $\mathrm{y}$, en lo posible, varias páginas o contenido en distintos archivos, debidamente identificados e identificables).

La exposición se lleva a cabo en una Sesión de Oratoria. Si es posible se trabaja en un auditorio, con micrófono, para que los formándose se vayan adaptando a sus espectadores. Se les pregunta si desean invitados o si, por lo contrario, solamente van a asistir los compañeros de Curso. Cuando no es posible trabajar en un auditorio, el salón de clase se ordena muy bien y se trata de adaptarlo a las condiciones y circunstancias de tal auditorio.

Cabe señalar que la etapa de DOCUMENTACIÓN, no necesariamente se ciñe a una exposición. También puede concretarse en un primer ensayo. Aclárase aquí que se les aconseja a los sujetos de investigación trabajar en equipo. Se les da, durante todo el proceso, 3 minutos por persona. Entonces, si el equipo es de, por citar un caso, 5 personas tendrán 15 minutos. Se aclara y explica que toda intervención debe comportar una presentación, un ejercicio de oratoria, el respectivo quehacer retórico, la fundamentación teórica del caso, y un final más inteligente que el principio. En efecto, desde la primera exposición o primer ensayo hasta la presentación definitiva se evalúa forma y fondo; es decir, oratoriaretórica y aportes teóricos.

EXPOSICIÓN: el tema elegido por los estudiantes $\mathrm{y}$ las respectivas realimentaciones son motivo de un trabajo académico denominado así. En tal momento, se explica el tema seleccionado en la etapa anterior. Gracias a la elicitación se ofrece ejemplos de presentaciones -con otros fundamentos teóricos, pero en el contexto retórico-, se discute con los estudiantes el porqué no se trata de darle gusto al profesor o de que a él le plazca lo presentado. Los principales interlocutores, se insiste a los estudiantes, son sus coetáneos. Por tal razón se parte del aforismo: "Lo bueno, si breve, dos veces bueno; y aun lo malo, si poco, no tan malo" (Baltasar Gracián). Además, se insiste en que los protagonistas de la Sesión de Retórica sean los primeros convencidos de la calidad de su presentación.

SEMINARIO ALEMÁN: esta etapa implica la discusión (en su acepción lingüística de llegar a acuerdos) sobre las presentaciones o ensayos preliminares; incluso, la misma teoría es puesta en escena de reflexión y de relatoría (en su esencia de tomar nota, con un fin específico, sobre documentos leídos previamente y entregados al relator; nunca se desarrolla una relatoría sobre hechos 
orales que no tengan, previamente, su correspondiente escrito). Aquí, el curso autocritica, critica, evalúa y proyecta acciones para despabilar a quienes aún no logran convencer, con su ensayo, al mismo grupo de compañeros; a quienes se nota incómodos en uno u otro papel; a quienes no están identificados con su propia presentación. Incluso, a aquellos que no logran la sincronía entre teoría y práctica o a presentaciones lineales, poco y nada prospectivas. También, en este momento vuelve a explicarse, si fuere necesario, nociones lingüísticas de difícil entendimiento. Verbi gratia, la relación monema, morfemas, palabras, oraciones, sintagmas, acto de habla, enunciado, entre otras.

INTERIORIZACIÓN-

ENCODIFICACIÓN: esta etapa es transversal; es producto del inicio del proceso, de su desarrollo y de su evolución. Debe reconocerse que los ensayos, generalmente de cuatro a cinco, coadyuvan la interiorización. $\mathrm{La}$ encodificación se da como pretexto y como sustancia de la Sesión de Retórica porque es allí en donde la teoría sobrepasa sus propios límites y se colige su utilidad. Es más, se justifica, en y desde la propia realidad, la inclusión de varios cursos de Linguiística en un currículo de Idiomas Modernos, dado que la ciencia del lenguaje es el asidero de la labor de quienes estudian la lengua como objeto, como dimensión, como esencia o como instrumento.

COEVALUACIÓN Y AUTOEVALUACIÓN: son estas las etapas finales del proceso y se dan luego de la puesta en escena -dramática, académica, descriptiva, narrativa, expositiva o argumentativa- de la Sesión de Retórica. Dado que el público es coetáneo con los protagonistas y que se trata de pares de otras Universidades, el profesor recoge las impresiones de los invitados (que, a propósito, son integrantes de otros de sus Cursos o de su Grupo de Investigación, o de su Grupo de Estudio) y las socializa con los estudiantes de Lingüística. Se expone lo bueno, lo virtuoso, lo imprevisto, lo persuasivo, lo oportuno, lo impertinente y lo superable. Lógicamente, sin entrar en el terreno de defender lo presentado y con la conciencia de que comunicar no es lo que se quiso decir -0 presentar en este caso- sino lo entendido y admirado, o no, por el público, los estudiantes justifican y enriquecen ciertos puntos de vista del público.

Valga citar aquí lo sucedido en la Sesión de Retórica motivo de estas líneas: su sede fue el Paraninfo de la UPTC. Era jueves en la noche. Infortunadamente, no se cuenta, luego de las dos primeras presentaciones, con la realización de una fiesta de una carrera upetecista, organizada en el patio central de la Alma Máter, con un grado extremo de ruido. Fiesta amenizada con música estridente y para todo tipo de escucha. La crítica de mayor ocurrencia es preguntarse el porqué no se empleó micrófono. Lógicamente, el público no sabe que uno de los derroteros del momento retórico es la equidad. Por tanto, si ya se había desarrollado dos intervenciones con voz en vivo, no podía darse otras condiciones a los otros grupos de trabajo. En este entendido, se encodifica en los estudiantes la necesidad de educar la voz, de aprovechar su máxima potencia, no sólo por ser futuro docente, sino porque no siempre se puede controlar variables ambientales cuando se organiza una actividad académica.

El recorrido anterior implica una observación semiestructurada. Mediante su cuaderno planeador de clase, el profesor toma nota, no sólo de la evaluación de cada 
ensayo, sino de características importantes connaturales con la Sesión de Retórica, a saber: un trabajo en equipo equitativo en donde se deje ver un quehacer no producto de distribuir obligaciones, sino de reunirse consuetudinariamente y con un fin común. Una participación de personajes o integrantes igualitaria tanto en tiempo como en protagonismo. No se permite que un integrante del grupo de trabajo lleve una responsabilidad o intervención de más de 30 segundos -recuérdese que son tres minutos por integrante- distribuidos en toda la presentación.

Un empleo pertinente y prudente de componentes lingüísticos: voz, tono y timbre; aforismos disciplinares, definiciones, conceptos e ideologías. Paralingüísticos: gestos, modismos, imitaciones, apropiaciones de un determinado papel, atuendos y, en general, uso de lengua o de habla según convenga, y extralinguiísticos: música, luces, elementos escenográficos, carteles, afiches, y elementos de tecnología blanda o tradicional, Cabe recordar aquí cómo cuando alguna presentación toca temas relacionados con discriminación, diferencias comportamentales, política, religiones, entre otros, se advierte la necesidad del respeto y de la prudencia para evitar estigmatizaciones a personas, a instituciones o a formas de vida.

\section{Corolarios}

La utilidad de la Sesión Interuniversitaria de Retórica, colegida a partir de la socialización, también interuniversitaria, de algunos contenidos propios de la Lingüística, en el contexto de un plan de estudios de Idiomas Modernos, como espacio de encodificación es notoria, conveniente, adecuada y trascendente porque:
Forma a los estudiantes desde la relación teoría-práctica. Les recuerda que la práctica sin teoría es descabellada y la teoría sin práctica es estéril. Entonces, la Academia se convierte en verdadero espacio de relación con lo real, lo social, lo común. Incluso, con lo laboral.

Acrecienta sus capacidades orales y de persuasión. Presentarse ante un público no es tarea fácil. Vencer los nervios para hablar ante coetáneos extraños no es sencillo. Cualidades de la expresión oral, como la mirada a los ojos, la dirección hacia el público, la voz adecuada con el local, entre otras, son parte sustancial de una Sesión Interuniversitaria de Retórica.

Indica la necesidad de cumplir procesos para llegar a logros contundentes. Gracias a las etapas elucidadas en la metodologá de este documento, los estudiantes se conciencian de que lo fundamental, en el desarrollo de una opción temática es afinar calidad con cantidad y es utilizar los conocimientos impartidos para aplicarlos en la solución de carencias propias del aula de clase. Carencias que, también, son parte de la vida misma.

Permite, a los futuros docentes, aplicar técnicas propias de una expresión oral académica y discernir características y diferencias de algunas metodologías -que, a la vez, son contenidos propios de un sillabus de Lingüística- propias de la enseñanza-aprendizaje de una lengua, a saber: la estructuralista, la formalista, la generativa, la discursista y la cognitiva.

Ayuda a entender la diferencia entre la lengua, como dimensión, como objeto de estudio, como instrumento y como esencia. Se comprueba que el hecho de ser hablante de una lengua o nativo en 
y de ella, no es condición ni calidad para enseñarla $\mathrm{o}$ para pretenderse lector $\mathrm{o}$ escritor. Una lección importante, en este estado de cosas, es la necesidad de estudiar la lengua, no sólo para poderla enseñar. También, para poderla escribir.

Enriquece, en lo que a los profesores concierne, metodologías investigativas. Se trata de fundamentar la experiencia con las lecturas y las escrituras propias de la misma. En este caso, con la ciencia del lenguaje. Con los años, la Sesión interuniversitaria de Retórica ha enseñado la prolijidad y la disimilitud conceptual de la ciencia del lenguaje, la naturaleza de los conceptos propios de la misma, el cambio de enfoque en cuanto el objeto de estudio de tal ciencia, los conceptos operativos de la misma, la necesidad de estudiar las dicotomías lingüísticas, los aportes de cada una de las escuelas lingüísticas del siglo $\mathrm{XX}$, la virtud y los desaciertos de distintos fundamentos escolásticos en relación con el lenguaje $\mathrm{y}$, en el contexto de estos parágrafos, la fundamentación requerida por quien pretenda enseñar o aprender una lengua.

No puede terminarse esta parte del escrito sin enlistar algunas dificultades circunstanciales que conlleva el proceso explicado. Infortunadamente, se nota un trabajo en equipo individualizado, ora porque los estudiantes tienden a repartirse los contenidos y los privilegian, como única preocupación del dicho proceso. Así mismo, tiende a encasillarse una Sesión de Retórica, como un espacio exclusivo de humor y de risa. Entonces, los estudiantes con problemas de timidez - que rayan en posible agorafobia no clínica- se retraen y abandonan el proceso. Argumentan no tener herramientas expresivas que fomenten el humor. Probablemente, ello se da por la antigüedad de esta estrategia metodológica en la opción temática de Lingüística y en otras dirigidas por el autor. Como bien se sabe, los actores del proceso tienen a mitificarlo en desmedro de su verdadero valor y fines lingüísticos.

\section{Referencias Bibliográficas}

CARDOSO, Karen y CASTAÑEDA, Érika. (2017). La retórica y su importancia en los procesos orales académicos. Electiva Interdisciplinar I, Redacción Científica, ejercicio de construcción de un artículo científico. Tunja: UPTC.

CASSANY, Daniel. (1999). Construir la escritura. Barcelona: Paidós.

Coloquio Continuado de Gramática Española y Redacción Científica, COGRED. Paneles: Lenguas de América y Homenaje a los cien años de la Lingüística. Tunja, UPTC, 2016, 2017.

BERNÁRDEZ, Enrique. (1999). ¿Qué son las lenguas? Madrid: Alianza, nueva edición.

MAHECHA, Miguel Ángel. (2005). Ferdinand de Saussure. Écrits de linguistique générale. Sección Reseñas de FORMA Y FUNCIÓN. Bogotá: Universidad Nacional de Colombia, Número 16; (diciembre, 2005), p. 310323.

MARTINET, André. (1970). Elementos de lingüística general. Madrid: Gredos, 1970.

ROSENBLAT, Ángel y SANABRIA, Antonio. (1968). Rectifique usted sus conocimientos gramaticales. Tunja: UptcEdiciones la Rana y el Águila. 
SANTIAGO G., Álvaro; CASTILlO P., Myriam; RUIZ V., Jaime. (2006). Lectura, metacognición y evaluación. 2a ${ }^{\mathrm{a}}$ Ed. Bogotá: Alejandría.
TORRES CRUZ, Doris L. (2012). La lectura y la escritura, un espacio para el mejoramiento de la calidad educativa y social. DOCTORADO EN LENGUAJE Y CULTURA. Lecciones \& Escrituras. Tunja: UPTC, Doctorado en Lenguaje y Cultura, Facultad de Ciencias de la Educación, enero-diciembre, 2011-2012, pp. 223-226.

VEYRAT RIGAT, Monserrat. Psicolinguiística. (1999). LÓPEZ, Ángel, SERRA, Enrique, HERNÁNDEZ, Carlos, PRUÑONOSA, Manuel, MORANT, Ricardo, PENADÉS, Inmaculada. Linguiística general y aplicada. Valencia: la Universidad, 1999. pp. 349-376 